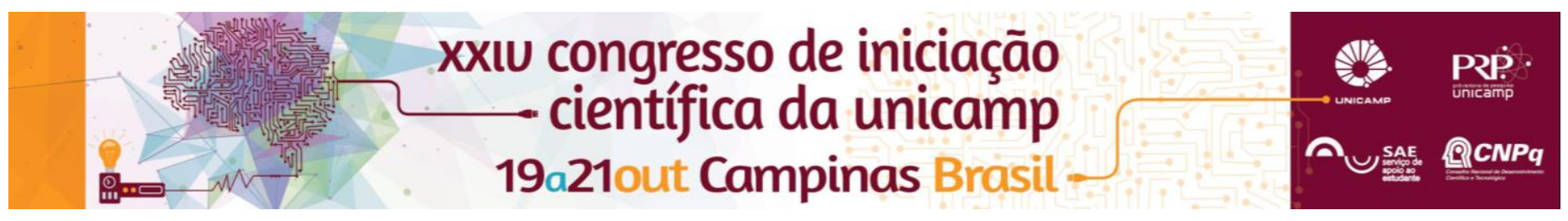

\title{
A streptomycete strain from the Caatinga Biome with potential herbicide activity
}

\author{
Luis G. Lantin*, Fábio S. P. Silva, Harold A. V. Hoyos, Itamar S. Melo
}

\begin{abstract}
The Caatinga is a semi-arid environment of the Brazilian territory. Despite the harsh conditions, high richness of species and high endemicity are found in this region. Based on this scope, we isolate actinobacteria from the Caatinga soil with herbicide activity. Thus, we selected an isolate P5.55 with strong herbicide activity. The organism shares a $16 \mathrm{~S}$ rRNA gene similarity of $98.07 \%$ with the type strain of S. enissocaesilis. We therefore indicated that the isolate P5.55 may represent a new species of the genus Streptomyces with great potential in agro biotechnology.
\end{abstract}

\section{Key words:}

Actinobacteria, Pre-emergence, Streptomyces sp.

\section{Introduction}

The Caatinga is a semi-arid environment of the Brazilan territory, covering an area of $841,261 \mathrm{~km}^{2}$, which corresponds about $11.0 \%$ of the country. Despite the harsh conditions, high richness of species and high endemicity is found in this region. Genomic studies of microbial diversity indicate that Actinobacteria are abundant in this environment ${ }^{1}$. Streptomyces is an important Actinobacteria genus and was first proposed by Waksman and Henrici (1943), and currently there are over 700 known species $^{2}$. A feature of the genus relies on the broad capacity of its members to produce bioactive molecule with biotechnological interest. The use of these microorganisms or their compounds in agriculture is still unimpressive, despite the synthetic agrochemicals are less efficient especially in herbicides sector because of weed resistance.

\section{Results and Discussion}

A serially diluted suspension of $1 \mathrm{~g}$ of soil collected in the Caatinga Biome was incubated at $28^{\circ} \mathrm{C}$ for 21 days. The colonies, morphologically similar to Streptomyces, were selected and purified on oatmeal agar plates (ISP 3) and subjected to bioassay for herbicide activity in Lactuca sativa. The isolate P5.55 was recovered from humic acid and vitamins agar plates (HVA) and was selected due to herbicidal activity (Figure 1).

The phylogenetic positon of the isolate P5.55 was determined by 16S rRNA gene sequence analysis. Sequencing was performed using a Genetic Analyzer 3500 sequencer (Applied Biosystems). The almost complete 16S rRNA gene sequence (1461 nucleotides [nt]) of isolate P5.55 was aligned using MEGA version 5.2 software against corresponding sequences of closely related type strains of Streptomyces species retrieved from the GenBank database using the EzTaxon-e server (Kim et al. 2012). Phylogenetic tree and support analysis ("bootstrap"; 1000 replicates) were inferred using three tree-making algorithms drawn from the MEGA version 5.2 package. Thus, the isolate P5.55 had a 16S rRNA gene similarity of $98.07 \%$ with the type strain of $S$. enissocaesilis NRRL B$16365^{\top}$, a value which corresponds to 28 different nucleotides at 1,452 sites (Figure 2).

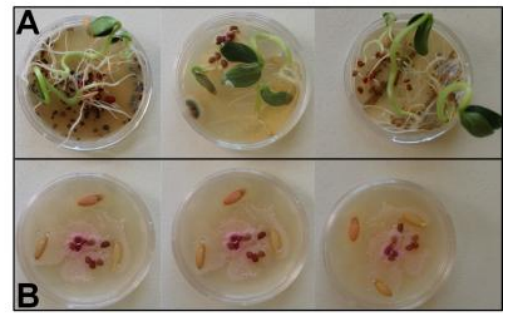

Figure 1. Pre-emergence herbicide activity observed in Lactuca sativa (dicot.). A-Control, B-Strain P5.55 inoculated

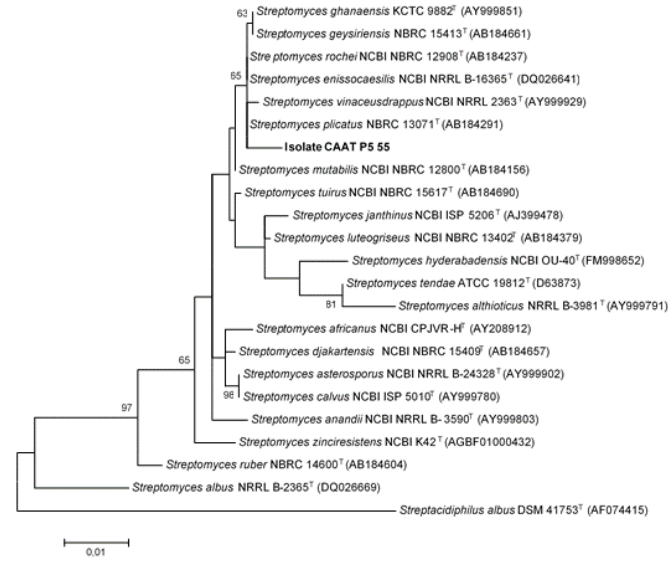

Figure 2. Neighbour-joining tree based on nearly complete 16S rRNA gene sequence showing the relationships between strain P5.55 and the type strains of phylogenetically closely related Streptomyces species.

\section{Conclusions}

The isolate P5.55 has herbicidal activity and may represent a new species of the genus Streptomyces due to low similarity of the 16S rRNA gene. Howerver, these results should be investigated with the taxonomic study for description of the strain.

\section{Acknowledgement}

We would like to acknowledge to EMBRAPA and CNPq. This work was supported by the CNPQ-PROTAX (grant number 163427/2015-1).

${ }^{1}$ Lançoni, M. D.; Taketani, R. G.; Kavamura, V. N e Melo, I. S. World J. Microbiol. Biotechnol. 2013, 29, 1233-1241.

${ }^{2}$ Labeda, D. P. et al. Antonie Van Leeuwenhoek. 2012, 101, 73-104 